\title{
Determinants of the Increase in Fasting Plasma Ketone Concentration during SGLT2 Inhibition in NGT, IFG and T2DM Patients
}

Hussein Al Jobori, MD, Giuseppe Daniele, MD, John Adams, MSc, Eugenio Cersosimo, MD, PhD, Curtis Triplitt, PharmD, Ralph A. DeFronzo, MD and Muhammad Abdul-Ghani, MD, PhD

Diabetes Division, University of Texas Health Science Center at San Antonio.

\author{
Keywords: SGLT2 inhibitor, empagliflozin, fasting plasma glucose, ketone, free fatty acid, \\ insulin, glucagon. \\ Running Title: empagliflozin and plasma ketone \\ Address all correspondence to: \\ Ralph A. DeFronzo, MD \\ Diabetes Division \\ University of Texas Health Science Center at San Antonio \\ 7703 Floyd Curl Drive \\ San Antonio, TX 78231 \\ Phone: 210-567-6691 \\ FAX: 210-567-6554 \\ Email: albarado@uthscsa.edu
}

\begin{abstract}
Aim: To examine metabolic factors that influence ketone production after sodium-glucose cotransport inhibitor (SGLT2) administration

Research Design and Methods: Fasting plasma glucose, insulin, glucagon, free fatty acid and ketone concentrations were measured in 15 type 2 diabetes mellitus (T2DM) and 16 non-diabetic subjects before and at 1 and 14 days after treatment with empagliflozin.
\end{abstract}

Results: Empagliflozin caused $38 \mathrm{mg} / \mathrm{dl}$ reduction in the fasting plasma glucose (FPG) concentration in T2DM patients. However, it caused only small but significant $(7 \mathrm{mg} / \mathrm{dl})$

This article has been accepted for publication and undergone full peer review but has not been through the copyediting, typesetting, pagination and proofreading process, which may lead to differences between this version and the Version of Record. Please cite this article as doi: 10.1111/dom.12881

This article is protected by copyright. All rights reserved. 
reduction in the FPG concentration in impaired fasting glucose (IFG) subjects and did not affect the FPG concentration in normal glucose tolerant (NGT) subjects. Empagliflozin caused a significant increase in the mean plasma glucagon, free fatty acid (FFA) and ketone concentrations in T2DM subjects. However, empagliflozin did not cause a significant change in the mean plasma insulin, glucagon or ketone concentrations in non-diabetic subjects. An index which integrates the change in plasma glucose, insulin and FFA concentration at day 1 strongly correlates with plasma ketone concentration at day $1(\mathrm{r}=0.85, \mathrm{p}<0.001)$ and day $14(\mathrm{r}=0.63$, $\mathrm{r}=0.01$ ) and predicts with $86 \%$ sensitivity and $83 \%$ specificity subjects at the top tertile for plasma ketone concentration after empagliflozin treatment.

Conclusion: The results of the present study demonstrate that SGLT2 inhibition exert different metabolic effects in non-diabetic individuals compared diabetic patients.

\section{INTRODUCTION}

Sodium-glucose co-transport (SGLT2) inhibitors are a novel class of antidiabetic drugs approved by the Food and Drug Administration for the treatment of T2DM (1). Members of this class of drugs lower the plasma glucose concentration by inhibiting renal glucose transport and producing glucosuria (1-2). Because of their unique mechanism of action, SGLT2 inhibitors are effective in lowering the plasma glucose concentration as monotherapy and in combination with all other antidiabetic agents including insulin (2). Recent reports have demonstrated that some subjects receiving SGLT2 inhibitors experience clinically significant ketoacidosis events despite being normoglycemic (3-5). These euglycemic ketoacidotic events following SGLT2 
administration were primarily reported in patients with type 1 diabetes $(3,4)$, and in type 2 diabetes as well (5).

In addition to lowering the plasma glucose concentration, SGLT2 inhibitors exert multiple other metabolic actions in T2DM individuals (6). They improve insulin sensitivity (7) and beta cell function (8), stimulate an increase in the basal rate of hepatic glucose production, lower fasting plasma insulin concentration, stimulate glucagon secretion $(7,9)$ and switch fuel consumption from glucose to fat oxidation $(9,10)$. Some of these metabolic actions of SGLT2 inhibitors can affect ketone production in T2DM patients. Recently, we (10) and others (11) have demonstrated that administration of SGLT2 inhibitors for 2-4 weeks in T2DM patients caused significant increase in fasting plasma ketone concentration. To examine factors that regulate glucose and fat oxidation and could lead to the increase in plasma ketone concentration in T2DM patients, we have compared the effect of empagliflozin treatment on plasma glucose, insulin, glucagon, free fatty acid (FFA) and ketone concentration in T2DM and non-diabetic individuals before and 1 and 14 days after treatment with empagliflozin.

\section{Research Design and Methods}

Subjects: 31 subjects who were part of study designed to examine the effect of empagliflozin on the kinetics of renal glucose handling participated in this study. 15 type 2 diabetic subjects, and 16 non-diabetic (8 with normal fasting glucose [FPG $<100 \mathrm{mg} / \mathrm{dl}]$; and 8 with impaired fasting glucose [FPG=100-125 mg/dl])subjects according to the American Diabetes Association criteria. All subjects were in good general health as determined by medical history, physical exam, screening lab tests, and EKG. Body weight was stable ( \pm 3 pounds) in all subjects for 3 months prior to study and no subject participated in any excessively heavy exercise program. Patients 
with T2DM were on a stable dose of metformin and/or sulfonylurea . None of the non-diabetic subject was taking any medication known to affect glucose metabolism.

After screening, eligible subjects received measurement of fasting plasma glucose (mean of 4 measurements), insulin (mean of 2 measurements), glucagon (mean of 2 measurements), free fatty acid (mean of 2 measurements) and ketone concentration (mean of 3 measurements) at baseline. On day 1, subjects were started on empagliflozin (25 mg/day) for 14 days. On day 1 and day 14, fasting plasma glucose, insulin, glucagon, free fatty acid and ketone concentrations were repeated. The study was approved by UTHSCSA Institutional Review Board and informed written consent was obtained from all participants before the start of the study.

\section{Analytical Methods}

Plasma glucose was measured by glucose oxidase method (Beckman Instruments, Fullerton, CA). Plasma insulin was measured by radioimmunoassay (Diagnostic Products Corp., Los Angeles, CA). Plasma FFA was determined by enzymatic colorimetric quantification method (Wako Chemicals, Nuess, Germany). Plasma ketone concentration (beta hydroxyl

butyrate and aceto-acetate) was measured by commercially available kit (Caymann Chemical, MI, USA).

\section{Calculations and statistical analysis}

The Ketogenic Index: to generate and index that predicts the risk of increase in plasma ketone concentration after empagliflozin administration, we examined the correlation, in the entire cohort, between plasma ketone concentration and plasma glucose concentration before and 1 day after empagliflozin treatment, plasma insulin, glucagon and FFA concentration at day 1 after empagliflozin treatment. Fasting plasma insulin and the decrease in plasma glucose 
concentration significantly and inversely correlated with plasma ketone concentration while plasma FFA concentration significantly correlated with plasma ketone concentration. Thus, we defined the Ketogenic Index $=\log \left[\mathrm{FFA} X(1 / \mathrm{FPI}) \mathrm{X}\left(\mathrm{FPG}_{\mathrm{pre}} / \mathrm{FPG}_{\text {treat }}\right)\right]$.

FFA= plasma free fatty acid concentration (in $\mathrm{mM}$ ) after starting treatment; FPI= fasting plasma insulin concentration $(\mu \mathrm{U} / \mathrm{ml})$ after starting treatment; $\mathrm{FPG}_{\mathrm{pre}}=$ fasting plasma glucose concentration (mg/dl) before starting treatment with empagliflozin; and $\mathrm{FPG}_{\text {treat }}=$ fasting plasma glucose concentration at day 1 after starting treatment with empagliflozin.

The addition of plasma glucagon concentration to the index did not significantly improve its correlation with plasma ketone concentration.

Values are presented as mean \pm S.E.M. The difference between means was compared with ANOVA. Correlation between variables was tested with Pearson's correlation. Significance was set at $\mathrm{p}<0.05$.

\section{RESULTS}

Table 1 presents clinical characteristics of study participants.

Empagliflozin caused $45 \pm 4,50 \pm 5$ and $97 \pm 11$ grams glucosuria per day in NGT, IFG and T2DM subjects, respectively. As anticipated, empagliflozin caused 26 and $38 \mathrm{mg} / \mathrm{dl}$ reduction in the FPG concentration at day 1 and day 14, respectively, (both $\mathrm{p}<0.0001$ ), in T2DM patients. Conversely, the fasting plasma glucose concentration in NGT subjects was not affected by empagliflozin treatment $(95 \mathrm{mg} / \mathrm{dl}$ vs 96 and $94 \mathrm{mg} / \mathrm{dl}$ before and at day 1 and 14 after treatment, 
respectively) despite 45 grams per day of urinary glucose loss. Although urinary glucose loss was comparable in IFG subjects and NGT subjects, empagliflozin caused a modest but significant reduction in the FPG concentration (from $110 \pm 2$ to $104 \pm 3 \mathrm{mg} / \mathrm{dl}, \mathrm{p}<0.01$ ) in IFG subjects. Further, the magnitude of decrease in the FPG concentration strongly and inversely correlated with the FPG concentration $(r=-0.80, \mathrm{p}<0.0001)$ (Figure 1) and this relationship was maintained in non-diabetic subjects $(\mathrm{r}=-0.61, \mathrm{p}<0.01)$.

As anticipated and consistent with previous studies (6-9), empagliflozin caused a significant decrease (by 27\%, $\mathrm{p}=0.05$ ) in the fasting plasma insulin concentration and a large increase in the fasting plasma glucagon concentration at day 1 in T2DM patients (Table 2). The increase in fasting plasma glucagon was maintained at day 14, however, the FPI concentration returned to the pretreatment level at day 14 . Conversely, empagliflozin did not significantly affect the fasting plasma insulin and glucagon concentrations in NGT and IFG subjects.

Fasting plasma FFA was $543 \pm 68,467 \pm 37$ and $740 \pm 57 \mu \mathrm{M}$ in NGT, IFG and T2DM patients respectively, and increased similarly in all 3 groups in day $1(\mathrm{p}=0.01)$, but returned back to the pretreatment level at day 14 in all 3 groups.

Consistent with previous studies $(10,11)$, empagliflozin caused a significant increase in fasting plasma ketone concentration in T2DM patients (from $528 \pm 36$ to $670 \pm 49 \mu \mathrm{M}$ in day $14, \mathrm{p}=0.03$ ), while plasma ketone concentration was not affected by empagliflozin treatment in either IFG (436 \pm 31 vs $421 \pm 32$ ) or NGT ( $537 \pm 64$ vs $451 \pm 32 \mu \mathrm{M}$ ) subjects following empagliflozin treatment (Figure 2).

\section{Relationship between plasma ketone, insulin, glucose, and FFA concentration}


The magnitude of decrease in plasma glucose concentration at day 1 did not significantly correlate with the change in plasma insulin, glucagon or FFA concentration at day 1. However, it significantly and inversely correlated with the plasma ketone concentration at day $1(\mathrm{r}=-0.63$, $\mathrm{p}<0.05)$ and the increase in plasma ketone concentration from baseline to day $1,(r=-0.74$, $\mathrm{p}=0.01$ ) (Figure 3). The plasma ketone concentration at day 1 strongly correlated with plasma FFA concentration at day $1(\mathrm{r}=0.59, \mathrm{p}<0.05)$ and inversely correlated with plasma insulin concentration at day $1(\mathrm{r}=-0.51, \mathrm{p}<0.05)$. As anticipated, the decrease in plasma insulin concentration was associated with an increase in plasma FFA concentration ( $\mathrm{r}=-0.55, \mathrm{p}=0.01)$. The ketogenic index strongly correlated with plasma ketone concentration at day 1 ( $\mathrm{r}=0.85$, $\mathrm{p}<0.001))$, day $14(\mathrm{r}=0.64, \mathrm{p}=0.01)$ and a cut point of -0.9 predicted subjects in the upper tertile (with plasma ketone concentration $>0.6 \mathrm{mM}$, mean $=0.76 \mathrm{mM}$ ) with $86 \%$ sensitivity and $83 \%$ specificity.

\section{DISCUSSION}

The present study has several novel observations. Unlike subjects with T2DM, emagliflozin did not affect the fasting plasma glucose concentration in NGT subjects despite large glucosuria (50 grams per day). Moreover, although glucosuria caused by empagliflozin in IFG was comparable to that in NGT, empagliflozin caused a small but significant decrease in the FPG concentration in IFG subjects. Further, the magnitude of decrease in the FPG concentration strongly and inversely correlated with the fasting plasma glucose concentration in the entire cohort and this relationship was maintained in non-diabetic individuals. The strong relationship between the

This article is protected by copyright. All rights reserved. 
magnitude of decrease in the FPG concentration and the FPG level in diabetic subjects can be explained by the fact that more glucose is removed from the body by SGLT2 inhibition as the FPG concentration increases and more glucose is filtered in the glumerolus (12). However, the differential effect of empagliflozin on the FPG concentration between IFG and NGT subjects can not be explained by differences in the amount of glucose removed by the kidney since it was comparable between both groups. Previous study with dapagliflozin in NGT subjects has reported no effect of the drug on the FPG concentration over 14 days treatment (13). These results suggest that it is likely that the decrease in FPG concentration in IFG subjects by empagliflozin is not simply due to urinary glucose loss, rather it is likely to correction of some of the underlying mechanisms responsible for the rise in FPG concentration in IFG subjects (14).

Although not measured in the present study, the basal rate of hepatic glucose production (HGP) has to increase following empagliflozin administration to compensate for the large urinary glucose loss in non-diabetic individuals and maintain the FPG concentration at euglycemia. A recent study (11) has reported even greater increase (32\%) in bHGP in non-diabetic than in diabetic (24\%) individuals following 4-week empagliflozin treatment. Nonetheless, unlike in T2DM patients, empagliflozin did not significantly affect the FPI or fasting plasma glucagon concentration in non-diabetic individuals. This finding is surprising and argues against an important role of increase in plasma glucagon concentration in the stimulation of bHGP in T2DM following SGLT2 administration in T2DM patients (7,9). Recent study (15) reported the presence of SGLT2 in alpha cell and suggested that inhibition of SGLT2 in the alpha cell with dapagliflozin strongly stimulates glucagon secretion. The results of the present study demonstrate that in the absence of change in the fasting plasma glucose concentration, e.g. in NGT individuals, empagliflozin did not affect glucagon secretion, suggesting that, at least in 
vivo in man, the decrease in the FPG concentration subsequent to urinary glucose loss plays more important role in simulating glucagon secretion than direct effect of the drug on the alpha cell.

The increase in plasma ketone concentration strongly and inversely correlated with the decrease in plasma glucose concentration. The increase in plasma ketone concentration also strongly correlated with plasma FFA concentration and inversely correlated with fasting plasma insulin concentration at day 1. Further, the ketogenic index strongly correlated with plasm ketone concentration in day 1 and day 14. The increase in fasting plasma FFA concentration explains, at least in part, the increase in fat oxidation caused by SGLT2 inhibitor which was reported in previous studies $(10,11)$ which could lead to increase in ketone production. Because insulin is very powerful inhibitor of lipolysis (16), the decrease in fasting plasma insulin explains the increase in plasma FFA concentration and indeed the increase in plasma FFA concentration strongly and inversely correlated with the decrease in plasma insulin concentration $(r=-0.55$, $\mathrm{p}=0.01$ ). These results suggest that the decrease in FPG concentration caused by glucosuria is likely the primary factor which triggers the other metabolic actions of SGLT2 inhibitors. The decrease in FPG concentration stimulates glucagon secretion and inhibits insulin secretion. Because of the powerful inhibitory effect of insulin on lipolysis, the decrease in FPI concentration results in an increase in plasma FFA and subsequent increase in fat oxidation and ketone production. Thus, the ketogenic index, which integrates the decrease in plasma glucose and insulin concentrations and increase in plasma FFA concentration, strongly correlated with plasma ketone concentration at day 1 and day 14 after empagliflozin administration, and predicted with high sensitivity and sensitivity subjects in whom the increase in plasma ketone concentration was in the top tertile. Thus, it will be of great interest to examine whether therapies 
which stimulate insulin secretion and decrease plasma FFA concentration, e.g. GLP-1 RA and pioglitazone, will mitigate the increase in ketone production and risk of ketoacidosis caused by SGLT2 administration.

In summary, the results of the present study demonstrate that it is likely that the change in plasma insulin and glucagon concentrations after SGLT2 is secondary to the decrease in FPG, and it does not play important role in stimulating HGP, but result in increase in FFA and stimulation of ketone production.

Acknowledgment: This study was supported in part by a grant from Boehringer-Ingelheim to RAD. We thank Sandra Martinez, RN and James King RN for their excellent care of the patients during the study and Lorrie Albarado and Shannon Balmer for their expert secretarial assistance in preparation of the manuscript. Dr. Ralph DeFronzo’s salary is supported, in part, by the South Texas Veterans Health Care System.

\section{REFERENCES}

1) Abdul-Ghani MA, Norton L, Defronzo RA: Role of sodium-glucose cotransporter 2 (SGLT 2) inhibitors in the treatment of type 2 diabetes. Endocr Rev 2011;32:515-531

2) Monica Reddy RP, Inzucchi SE. SGLT2 inhibitors in the management of type 2 diabetes. Endocrine. 53: 364-72, 2016.

This article is protected by copyright. All rights reserved. 
3) Modi A, Agrawal A, Morgan F Euglycemic Diabetic Ketoacidosis. Curr Diabetes Rev. 2016 [Epub ahead of print]

4) Peters AL, Buschur EO, Buse JB, Cohan P, Diner JC, Hirsch IB Euglycemic Diabetic Ketoacidosis: A Potential Complication of Treatment With Sodium-Glucose Cotransporter 2 Inhibition. Diabetes Care. 38:1687-93, 2015.

5) Peters AL, Henry RR, Thakkar P, Tong C, Alba M. Diabetic Ketoacidosis With Canagliflozin, a Sodium-Glucose Cotransporter 2 Inhibitor, in Patients With Type 1 Diabetes. Diabetes Care. 39: 5328, 2016

6) DeFronzo RA, Norton L, Abdul-Ghani M. SGLT2: Renal, Metabolic, \& Cardiovascular Considerations. Nature Rev Neph. 2016 (in press)

7) Merovci A, Solis-Herrera C, Daniele G, Roy Eldor R, Fiorentino T, Tripathy D, Xiong J, Perez Z, Norton L, Abdul-Ghani M, DeFronzo R. Dapagliflozin improves muscle insulin sensitivity but enhances glucose production. J. Clin Invest. 124:509-514, 2014

8) Merovci A, Abdul-Ghani M, Mari A, Solis C, Xiong J, Daniele G, Chavez A, Tripathy D, McCarthy SU, DeFronzo RA. Dapagliflozin Lowers Plasma Glucose Concentration and Improves Beta Cell Function. J Clin Endo Metab, 100:1927-32, 2015

9) Ferrannini E, Baldi S, Frascerra S, Astiarraga B, Heise T, Bizzotto R, Mari A, Pieber TR, Muscelli E Shift to Fatty Substrate Utilization in Response to Sodium-Glucose Cotransporter 2 Inhibition in Subjects Without Diabetes and Patients With Type 2 Diabetes. Diabetes. 65:1190-5, 2016

10) Daniele G, Xiong J, Solis-Herrera C, Merovci A, Eldor R, Tripathy D, DeFronzo RA, Norton L, Abdul-Ghani M Dapagliflozin Enhances Fat Oxidation and Ketone Production in Patients With Type 2 Diabetes. Diabetes Care. 2016 [Epub ahead of print]

This article is protected by copyright. All rights reserved. 
11) Ferrannini E, Muscelli E, Frascerra S, Baldi S, Mari A, Heise T, Broedl UC, Woerle HJ. Metabolic response to sodium-glucose cotransporter 2 inhibition in type 2 diabetic patients. J Clin Invest. 124:499-508, 2014

12) Abdul-Ghani MA, DeFronzo RA, Norton L.. Novel hypothesis to explain why SGLT2 inhibitors inhibit only 30-50\% of filtered glucose load in humans. Diabetes. 62:3324-8, 2013

13) Komoroski B, Vachharajani N, Boulton D, Kornhauser D, Geraldes M, Li L, Pfister M. Dapagliflozin, a novel SGLT2 inhibitor, induces dose-dependent glucosuria in healthy subjects. Clin Pharmacol Ther. 85: 520-6, 2009.

14) Alatrash M, Agyin C, Adams J, DeFronzo RA, Abdul-Ghani M, Decreased basal hepatic glucose uptake in subjects with impaired fasting glucose. Diabetologia 2016 (in press).

15) Bonner C, Kerr-Conte J, Gmyr V, Queniat G, Moerman E, Thévenet J, Beaucamps C, Delalleau N, Popescu I, Malaisse WJ, Sener A, Deprez B, Abderrahmani A, Staels B, Pattou F. Inhibition of the glucose transporter SGLT2 with dapagliflozin in pancreatic alpha cells triggers glucagon secretion. Nat Med. 21:512-7, 2015

16) Groop LC, Bonadonna RC, Del Prato S, Ratheiser K, Zyck K, Ferrannini E, DeFronzo RA Glucose and free fatty acid metabolism in non-insulin-dependent diabetes mellitus. Evidence for multiple sites of insulin resistance. J Clin Invest. 84:205-13, 1989.

Figures Legends:

This article is protected by copyright. All rights reserved. 
Figure 1: relationship between the decrease in fasting plasma glucose concentration at day 14 by empagliflozin and the fasting plasma glucose concentration before the start of treatment

Figure 2: Plasma ketone concentration before, at day 1 and day 14 after treatment with empagliflozin in NGT, IFG and T2DM patients

Figure 3: Relationship between the change in plasma glucose concentration and change in plasma ketone concentration after treatment with dapagliflozin in NGT, IFG and T2DM patients

Figure 4: Relationship between the ketogenic index and plasma ketone concentration at day 1 after treatment with empagliflozin in in NGT, IFG and T2DM patients. 
Table 1: Baseline patient characteristics

\begin{tabular}{|l|c|c|c|c|}
\hline & NFG & IFG & T2DM & P \\
\hline Age (years) & $58 \pm 2$ & $59 \pm 3$ & $55 \pm 2$ & NS \\
\hline Gender (m/f) & $6 / 2$ & $5 / 3$ & $12 / 3$ & NS \\
\hline BMI $\left(\mathrm{kg} / \mathrm{m}^{2}\right)$ & $27.0 \pm 1.1$ & $30.3 \pm 0.8$ & $31.1 \pm 2.1$ & 0.04 \\
\hline FPG (mg/dl) & $95 \pm 2$ & $110 \pm 2$ & $195 \pm 9$ & $<0.0001$ \\
\hline HbA1c (\%) & $5.6 \pm 0.1$ & $5.7 \pm 0.1$ & $7.8 \pm 0.2$ & $\mathrm{P}<0.0001$ \\
\hline $\begin{array}{l}\text { Plasma Creatinine } \\
\text { (mg/dl) }\end{array}$ & $0.70 \pm 0.05$ & $0.79 \pm 0.04$ & $0.8 \pm .005$ & NS \\
\hline $\begin{array}{l}\text { eGFR (ml/min.1.73 } \\
\left.\mathrm{m}^{2}\right)\end{array}$ & $120 \pm 10$ & $99 \pm 6$ & $107 \pm 7$ & NS \\
\hline
\end{tabular}

This article is protected by copyright. All rights reserved. 
Table 2: Fasting plasma glucose, insulin, glucagon and FFA before, and at day 1 and day 14 after treatment with empagliflozin.

\begin{tabular}{|c|c|c|c|c|c|c|c|c|c|c|c|c|}
\hline & \multicolumn{3}{|c|}{ NGT } & \multicolumn{3}{|c|}{ IFG } & \multicolumn{3}{|c|}{ Non-Diabetic (IFG+NGT) } & \multicolumn{3}{|c|}{ T2DM } \\
\hline & Baseline & Day 1 & Day 14 & Baseline & Day 1 & Day 14 & Baseline & Day 1 & Day 14 & Baseline & Day 1 & Day 14 \\
\hline$F$ & $95 \pm 1$ & $96 \pm 3$ & $94 \pm 3$ & $110 \pm 2$ & $105 \pm 3 *$ & $104 \pm 3 *$ & $103 \pm 2$ & $101 \pm 2$ & $99 \pm 3$ & $195 \pm 9$ & $169 \pm 11^{*}$ & $157 \pm 8 *$ \\
\hline$\mu \mathrm{U} / \mathrm{ml})$ & $9 \pm 2$ & $9 \pm 2$ & $12 \pm 3$ & $19 \pm 2$ & $18 \pm 2$ & $17 \pm 2$ & $14 \pm 2$ & $14 \pm 2$ & $15 \pm 2$ & $15 \pm 3$ & $11 \pm 2^{*}$ & $15 \pm 2$ \\
\hline Glucagon & $69 \pm 2$ & $75 \pm 6$ & $74 \pm 9$ & $86 \pm 5$ & $97 \pm 9$ & $79 \pm 6$ & $78 \pm 5$ & $86 \pm 6$ & $77 \pm 5$ & $94 \pm 12$ & $120 \pm 19 *$ & $106 \pm 11^{*}$ \\
\hline FFA $(\mu M)$ & $543 \pm 68$ & $650 \pm 88$ & $417 \pm 52$ & $467 \pm 37$ & $615 \pm 90$ & $491 \pm 41$ & $504 \pm 40$ & $633 \pm 63^{*}$ & $454 \pm 63$ & $740 \pm 57$ & $885 \pm 42 *$ & $762 \pm 47$ \\
\hline Ketone $(\mu \mathrm{M})$ & $537 \pm 64$ & $492 \pm 40$ & $451 \pm 32$ & $436 \pm 31$ & $443 \pm 29$ & $421 \pm 32$ & $487 \pm 38$ & $468 \pm 26$ & $437 \pm 22$ & $528 \pm 36$ & $667 \pm 49 *$ & $670 \pm 100 *$ \\
\hline
\end{tabular}

$* \mathrm{P}<0.05$

This article is protected by copyright. All rights reserved. 


\section{Figure 1}

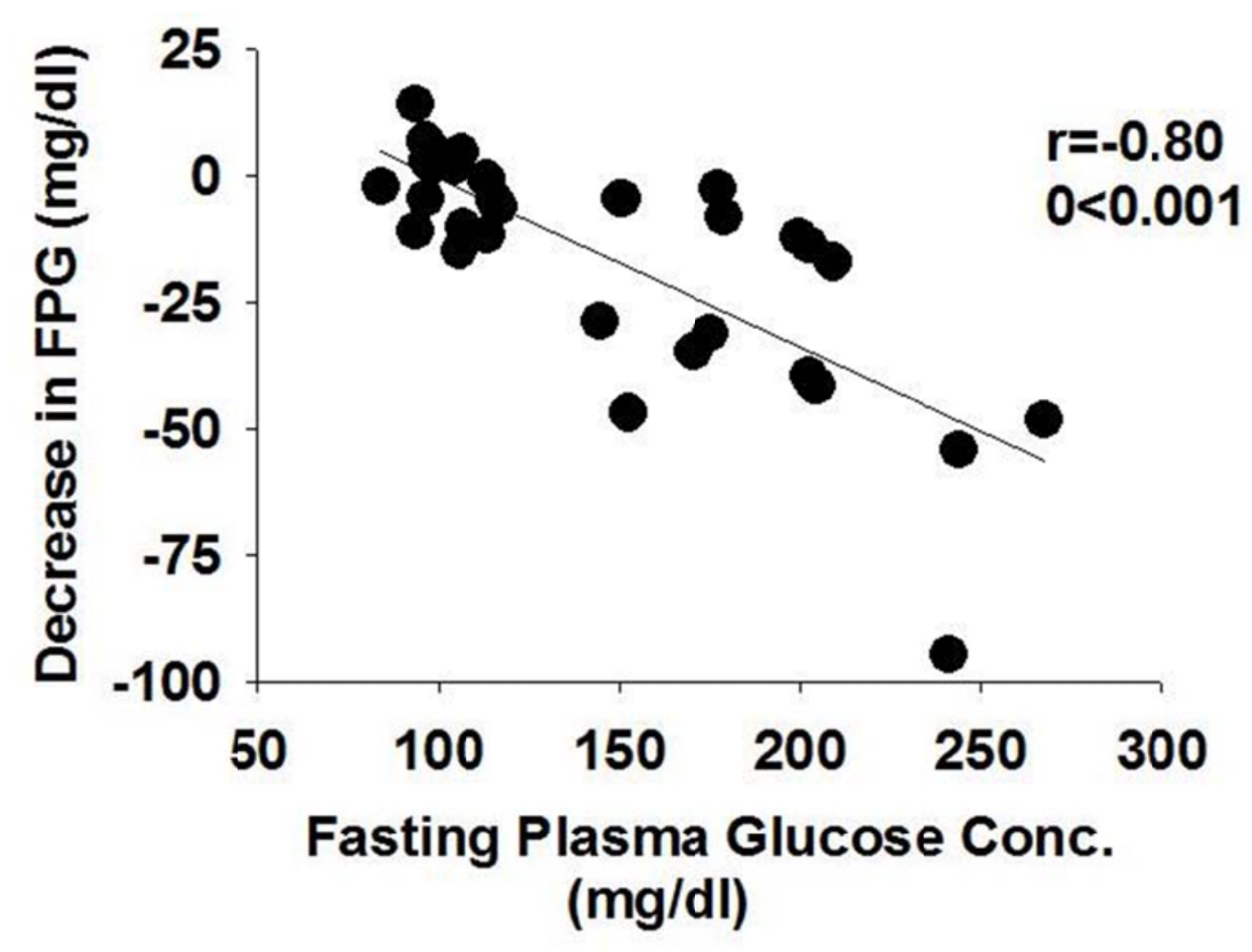




\section{Figure 2}

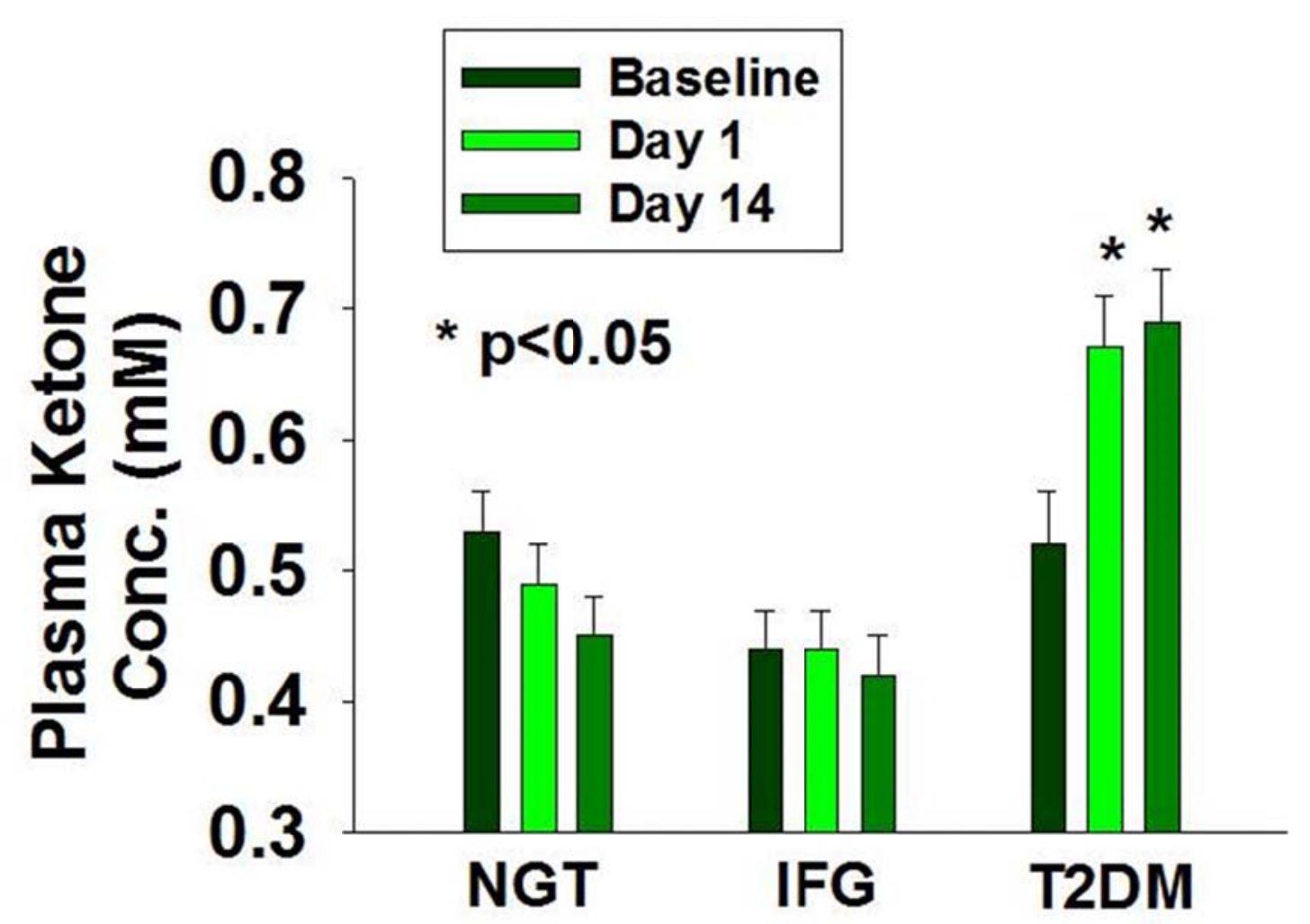




\section{Figure 3}

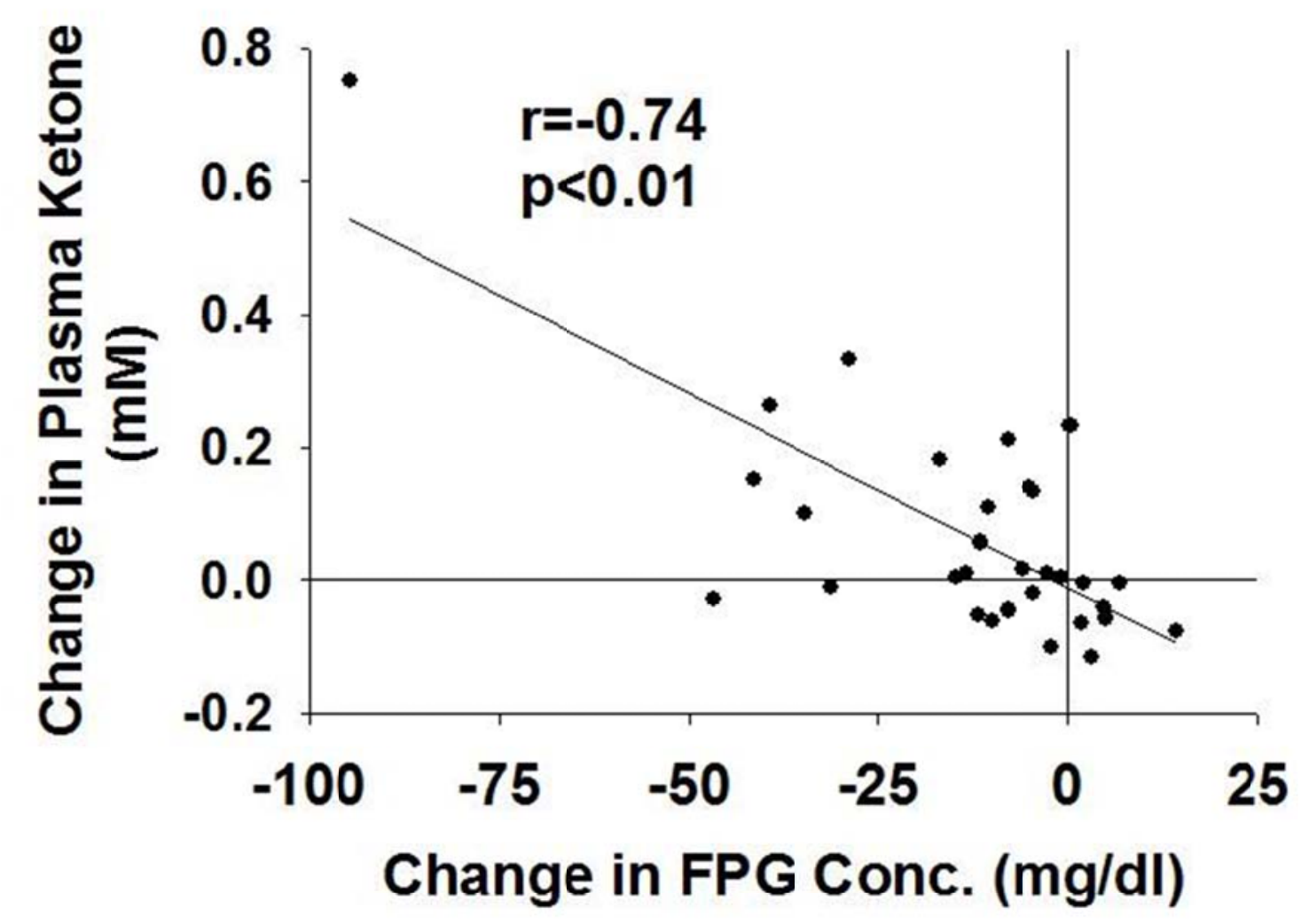

